\title{
Recurring sets of recurring starspot occultations on exoplanet host Qatar-2
}

\author{
T. Močnik, „ J. Southworth and C. Hellier \\ Astrophysics Group, Keele University, Staffordshire, ST5 5BG, UK
}

Accepted 2017 June 20. Received 2017 June 19; in original form 2016 August 26

\begin{abstract}
We announce the detection of recurring sets of recurring starspot occultation events in the short-cadence $K 2$ light curve of Qatar-2, a K dwarf star transited every $1.34 \mathrm{~d}$ by a hot Jupiter. In total, we detect 34 individual starspot occultation events, caused by five different starspots, occulted in up to five consecutive transits or after a full stellar rotation. The longest recurring set of recurring starspot occultations spans over three stellar rotations, setting a lower limit for the longest starspot lifetime of $58 \mathrm{~d}$. Starspot analysis provided a robust stellar rotational period measurement of $18.0 \pm 0.2 \mathrm{~d}$ and indicates that the system is aligned, having a skyprojected obliquity of $0^{\circ} \pm 8^{\circ}$. A pronounced rotational modulation in the light curve has a period of $18.2 \pm 1.6 \mathrm{~d}$, in agreement with the rotational period derived from the starspot occultations. We tentatively detect an ellipsoidal modulation in the phase curve, with a semiamplitude of $18 \mathrm{ppm}$, but cannot exclude the possibility that this is the result of red noise or imperfect removal of the rotational modulation. We detect no transit-timing and transitduration variations with upper limits of $15 \mathrm{~s}$ and $1 \mathrm{~min}$, respectively. We also reject any additional transiting planets with transit depths above $280 \mathrm{ppm}$ in the orbital period region $0.5-30 \mathrm{~d}$.
\end{abstract}

Key words: stars: fundamental parameters - stars: individual: Qatar-2 - planetary systems starspots.

\section{INTRODUCTION}

An obliquity is a misalignment angle between the stellar rotational and planet's orbital axis and is a key tracer of planet migration. Sky-projected obliquity can be measured spectroscopically by the Rossiter-McLaughlin effect (Gaudi \& Winn 2007), and by Doppler tomography (Collier Cameron et al. 2010) if a system is bright enough, or photometrically by tracing starspot occultation events (Sanchis-Ojeda \& Winn 2011). According to the Transiting Extrasolar Planets Catalogue (TEPCat) ${ }^{1}$ (Southworth 2011), sky-projected obliquity has been measured for a total of 100 systems, of which 85 host a transiting hot Jupiter (defined here as planets with orbital periods shorter than $10 \mathrm{~d}$ and masses between 0.3 and $13 M_{\text {Jup }}$ ). The discovery of the first hot Jupiter in 1995 triggered suggestions that such planets cannot form so close to their host stars but have instead formed beyond the ice line and migrated inwards to their current orbits (Mayor \& Queloz 1995). The planet formation and migration theories are far from well understood and expanding the sample of systems with known obliquities is of particular interest.

^E-mail: t.mocnik@keele.ac.uk

${ }^{1} \mathrm{http} / / / \mathrm{www}$.astro.keele.ac.uk/jkt/tepcat/tepcat.html
When a transiting planet occults a starspot it produces a bump of temporary brightening in the light curve (Silva 2003). The ability to detect starspot occultations is dependent on sufficient photometric precision and cadence of the observations, and on whether starspots are underneath a transit chord. Depending on the obliquity of the system the same starspot may be occulted in several consecutive transits or even after several stellar rotations.

Starspot occultations also provide a measurement of the stellar rotational period and add to our knowledge of starspots' lifetimes on stars other than Sun. Starspots' lifetimes are believed to be correlated with spot sizes and anticorrelated with supergranulae sizes (Bradshaw \& Hartigan 2014). In the case of the Sun the sunspots' lifetimes range from hours to several months, with a median lifetime of less than a day (Solanki 2003), and a longest recorded lifetime of an individual sunspot of $137 \mathrm{~d}$ (McIntosh 1981). On other stars the longest reported starspot lifetime using the occultation technique is $100 \mathrm{~d}$ for the active main-sequence star Kepler-17 (Désert et al. 2011), based on nearly continuous 17 months of Kepler observations (Borucki et al. 2010).

Since the failure of Kepler's second reaction wheel, its successor, $K 2$, suffers a reduced photometric precision due to the degraded pointing accuracy (Howell et al. 2014). Despite the shortcomings, $K 2$ continues to be able to detect starspots, as demonstrated by the 
detection of recurring starspot occultation events in the WASP-85 system (Močnik et al. 2016b).

Qatar-2 (2MASS J13503740-0648145, EPIC 212756297) is a moderately bright $V=13.3 \mathrm{~K}$ dwarf, hosting a hot Jupiter in a 1.34-d orbit, which was discovered by Bryan et al. (2012). Multicolour photometry of five transits by Mancini et al. (2014) revealed starspot occultations in all light curves with one potential occultation recurrence. The recurring occultation pair indicated a sky-projected obliquity of $4.3^{\circ} \pm 4.5^{\circ}$. The rotational period was reported as $11.4 \pm 0.5 \mathrm{~d}$, which has since been corrected to $14.8 \pm 0.3 \mathrm{~d}$ due to a calculation error (Mancini et al. 2016). However, the analysis presented in this paper proves that the two starspot occultations analysed by Mancini et al. $(2014,2016)$ were in fact caused by two different starspots and that even their corrected rotational period is incorrect.

In this paper, we use the $K 2$ short-cadence observations of Qatar-2, which reveals recurring sets of recurring spot crossings. Starspot analysis provided a robust determination of the stellar rotational period and a measurement of the sky-projected obliquity. We also measured the period of the pronounced rotational modulation, refined the system parameters, searched for transit-timing (TTVs) and transit-duration variations (TDVs), additional transiting planets and phase-curve variations. Lastly, we discuss the discrepancy between gyrochronological and isochronal age estimates.

After initial submission of this paper, Dai et al. (2017) announced a similar paper analysing the same $K 2$ data set. Their conclusions are largely in agreement with ours.

\section{K2 OBSERVATIONS}

Qatar-2 was observed during K2's Campaign 6 between 2015 July 14 and 2015 September 30 in the 1-min short-cadence observing mode. A continuous 79-d monitoring provided a total of 115890 images. We retrieved the target pixel file via the Minkulski Archive for Space Telescopes (MAST). The photometric extraction and spacecraft-drift artefact removal was carried out as done in Močnik et al. (2016b). In short, we used a 36-pixel fixed photometric aperture mask, temporarily removed the low-frequency stellar variability by normalizing the light curve with a low-order polynomial, and applied a self-flat-fielding (SFF) procedure using the Gaussian convolution of the measured normalized flux versus drift arclength. We then reintroduced low-frequency stellar variability by multiplying the corrected light curve with the same normalization polynomial as used earlier. The applied SFF procedure improved the median 1-min photometric precision from 1271 parts per million ( $\mathrm{ppm}$ ) to $854 \mathrm{ppm}$, which is within 10 per cent of the original Kepler precision for a similarly bright star. After removal of all the quality-flagged data points, such as thruster firing events and cosmic rays, we retained 111807 data points. The light curve before and after the SFF procedure is shown in Fig. 1.

\section{SYSTEM PARAMETERS}

To determine the system parameters, we performed a simultaneous Markov chain Monte Carlo (MCMC) analysis of the K2 photometry coupled with the Tillinghast Reflector Echelle Spectrograph (TRES) radial velocity measurements of Qatar-2 from Bryan et al. (2012). We re-applied the barycentric correction for TRES radial velocities to account for the issue that has been raised in their erratum where the original barycentric correction was calculated using the wrong declination. The corrected radial velocity measurements

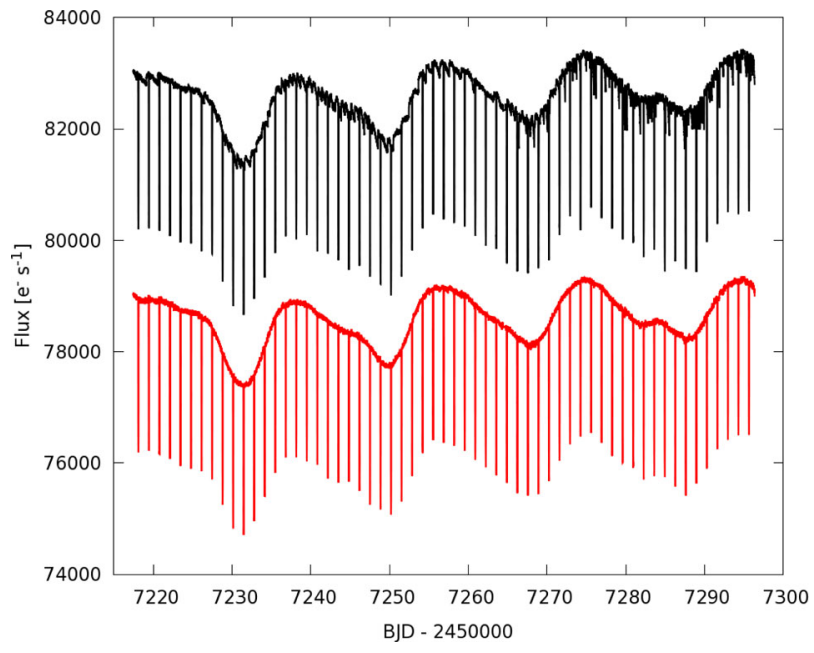

Figure 1. Binned short-cadence light curve of Qatar-2 before (shown in black) and after the SFF correction (red). A 10-min binning was used for this plot to reduce the white noise and to display the drift artefacts more clearly. The light curve exhibits a total of 59 transits and a pronounced starspot rotational modulation. The corrected light curve is shown with an offset of $-4000 \mathrm{e}^{-1} \mathrm{~s}^{-1}$ for clarity.

are available in Appendix A. The MCMC code is presented in Collier Cameron et al. (2007) and further described in Pollacco et al. (2008) and Anderson et al. (2015). Prior to the MCMC analysis, we removed the starspot occultation events from the $K 2$ light curve to prevent the potentially inaccurate parameter determination as discussed in Oshagh et al. (2013). We accounted for limb darkening by interpolating through tables of four-parameter limb-darkening coefficients, calculated for the Kepler bandpass by Sing (2010).

Because hot Jupiters are expected to circularize on a time-scale less than their age, we imposed a circular orbit to obtain the most likely parameters (e.g. Anderson et al. 2012). To estimate the upper limit on eccentricity, we ran a separate MCMC analysis with eccentricity being fitted as a free parameter and checked the eccentricity distribution in the resulting MCMC chain.

To improve the precision of the orbital period, we produced a separate MCMC analysis that also included the ground-based photometry presented in Bryan et al. (2012), with limb-darkening coefficients from Claret (2000, 2004), as appropriate for different bandpasses. This extended the photometric baseline from $79 \mathrm{~d}$ to $5.5 \mathrm{yr}$ and reduced the uncertainty to one fifth of its former value.

Table 1 lists the obtained system parameters and Fig. 2 shows the corresponding transit model.

We find a very good agreement with the system parameter values reported by Bryan et al. (2012) with a significantly improved precision. Mancini et al. (2014) reported stellar $(1.591 \pm 0.016 \rho \odot)$ and planetary densities $\left(1.183 \pm 0.026 \rho_{\text {Jup }}\right)$ significantly lower than Bryan et al. (2012). We find no evidence for the lower densities, as our values agree with those reported by Bryan et al. (2012).

\section{TTV AND TDV}

Measurements of mid-transit times and their deviations from a linear period ephemeris (transit-timing variations, or TTVs) can reveal additional and otherwise unobservable planets in planetary systems due to the inter-planet gravitational interactions (Agol et al. 2005). Reported TTV amplitudes for perturbed transiting exoplanets are between a few tens of seconds and several hours, with typical periods of the order of a few hundred days (Mazeh et al. 2013). Similarly, any 
Table 1. MCMC system parameters for Qatar-2 and Qatar-2b.

\begin{tabular}{|c|c|c|c|}
\hline Parameter & Symbol & Value & Unit \\
\hline Transit epoch & $t_{0}$ & $2457250.2008155 \pm 0.0000084$ & BJD \\
\hline Orbital period & $P$ & $1.33711677 \pm 0.00000010$ & $\mathrm{~d}$ \\
\hline Area ratio & $\left(R_{\mathrm{p}} / R_{\star}\right)^{2}$ & $0.026587 \pm 0.000062$ & - \\
\hline Transit width & $t_{14}$ & $0.075409 \pm 0.000046$ & $\mathrm{~d}$ \\
\hline Ingress and egress duration & $t_{12}, t_{34}$ & $0.010696 \pm 0.000064$ & $\mathrm{~d}$ \\
\hline Impact parameter & $b$ & $0.115 \pm 0.023$ & - \\
\hline Orbital inclination & $i$ & $88.99 \pm 0.20$ & $\circ$ \\
\hline Orbital eccentricity & $e$ & 0 (adopted; $<0.05$ at $2 \sigma$ ) & - \\
\hline Orbital separation & $a$ & $0.02136 \pm 0.00024$ & $\mathrm{au}$ \\
\hline Stellar mass & $M_{\star}$ & $0.727 \pm 0.024$ & $\mathrm{M}_{\odot}$ \\
\hline Stellar radius & $R_{\star}$ & $0.7033 \pm 0.0080$ & $\mathrm{R}_{\odot}$ \\
\hline Stellar density & $\rho_{\star}$ & $2.090 \pm 0.015$ & $\rho \odot$ \\
\hline Planet mass & $M_{\mathrm{p}}$ & $2.466 \pm 0.062$ & $M_{\text {Jup }}$ \\
\hline Planet radius & $R_{\mathrm{p}}$ & $1.115 \pm 0.013$ & $R_{\mathrm{Jup}}$ \\
\hline Planet density & $\rho_{\mathrm{p}}$ & $1.776 \pm 0.034$ & $\rho_{\text {Jup }}$ \\
\hline Planet equilibrium temperature ${ }^{a}$ & $T_{\mathrm{p}}$ & $1285 \pm 16$ & $\mathrm{~K}$ \\
\hline RV semi-amplitude & $K_{1}$ & $0.5609 \pm 0.0063$ & $\mathrm{~km} \mathrm{~s}^{-1}$ \\
\hline Limb-darkening coefficients & $a_{1}, a_{2}, a_{3}, a_{4}$ & $0.703,-0.737,1.486,-0.642$ & - \\
\hline
\end{tabular}

Note. ${ }^{a}$ Planet equilibrium temperature is based on assumptions of zero Bond albedo and complete day-to-night heat redistribution.

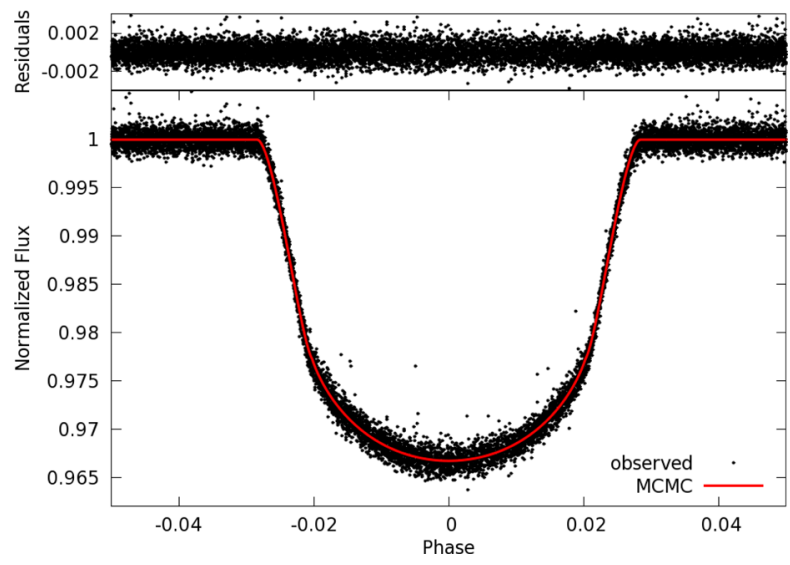

Figure 2. Best-fitting MCMC transit model and its residuals from the phasefolded $K 2$ light curve.

additional perturbing planet would also cause TDVs of the perturbed transiting planet, in phase with TTVs but with a significantly smaller amplitude (Nesvorný et al. 2013).

We measured the transit-timings and transit-durations for Qatar$2 \mathrm{~b}$ for each of the transits using the MCMC analysis as described in Section 3. Because the stellar activity can lead to inaccurate measurements of timings and durations (Oshagh et al. 2013), we, as in Section 3, removed the detected starspot occultations from the light curve prior to the MCMC analysis. The resulting TTV and TDV measurements are shown in Fig. 3. The data point near BJD 2457269 has been excluded from the analysis due to the qualityflagged data gap (see transit number 39 in Fig. 5).

The TTV and TDV measurements yield $\chi^{2}$ values of 63.0 and 55.5 , respectively, for 58 degrees of freedom, which means that they are statistically consistent with the assumption of white noise distributed around zero. We estimate the semi-amplitude upper limit to be $15 \mathrm{~s}$ for TTVs and $1 \mathrm{~min}$ for TDVs. The absence of TTVs and TDVs indicates that a second, non-transiting, massive planet is unlikely, unless at a much longer period.
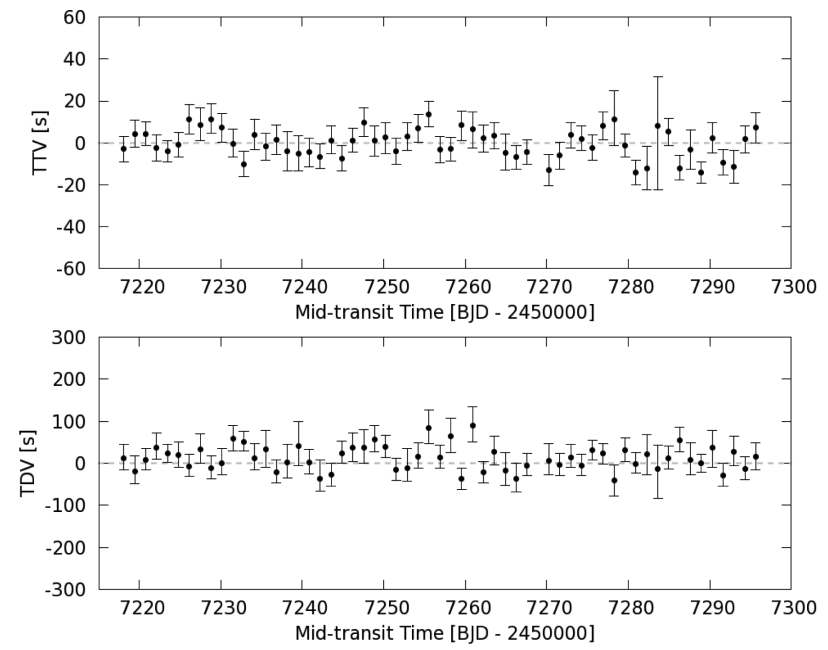

Figure 3. The TTV (upper panel) and TDV (lower panel) measurements are statistically consistent with the assumption of strictly periodic transits and constant transit duration.

\section{ROTATIONAL MODULATION}

The K2 light curve of Qatar-2 exhibits a pronounced rotational modulation with an amplitude of about 2 per cent (see Fig. 1). The modulation is caused by the presence of starspots on the surface of the rotating host star. To measure the period, we first removed the transits from the light curve, applied $10 \sigma$ clipping and then calculated a Lomb-Scargle periodogram. The rotational modulation period is manifested as the highest peak near $18.2 \mathrm{~d}$ with a Gaussian standard deviation of $1.6 \mathrm{~d}$ (see Fig. 4).

The resulting rotational modulation period of $18.2 \pm 1.6 \mathrm{~d}$ is a direct measure of the rotational period. We measure a similar rotational period well within the uncertainty also when using three publicly available drift-corrected long-cadence light curves (K2SFF Vanderburg \& Johnson 2014, K2SC Aigrain, Parviainen \& Pope 2016 and EVEREST Luger et al. 2016), which dismisses any doubts of whether our custom artefact removal technique might contaminate the rotational modulation. 


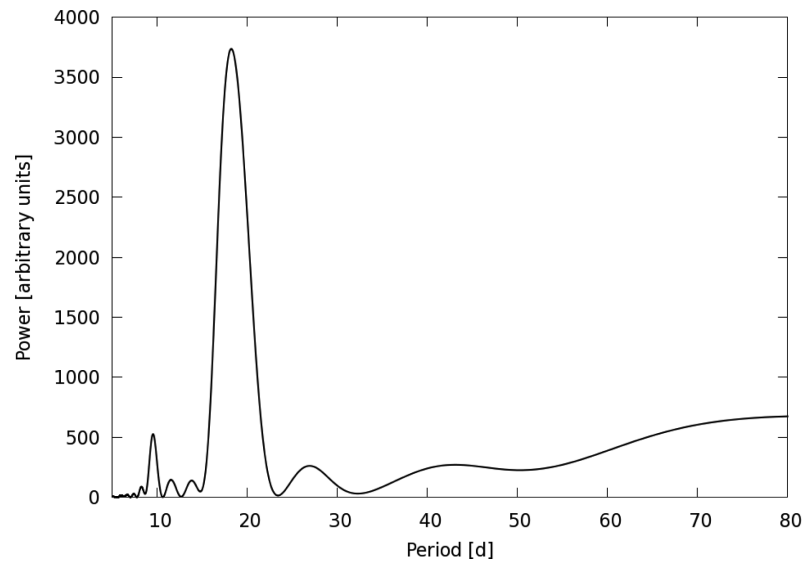

Figure 4. Lomb-Scargle periodogram of the rotational modulation. The highest peak suggests the rotational period of $18.2 \pm 1.6 \mathrm{~d}$.
The stellar rotational period given above and stellar radius given in Table 1 correspond to a stellar equatorial rotational velocity of $2.0 \pm 0.2 \mathrm{~km} \mathrm{~s}^{-1}$, whereas the spectroscopically measured stellar projected rotational velocity is $2.0 \pm 1.0 \mathrm{~km} \mathrm{~s}^{-1}$ (Esposito et al. 2017). Although the calculated rotational velocity and the measured projected rotational velocity match, suggesting an edgeon stellar rotation, we can constrain the inclination only very weakly as being larger than $30^{\circ}$ owing to a large relative error bar for the projected rotational velocity. A stronger indication for a near-edgeon stellar rotation is revealed by the starspot position measurements in Section 6.3.

\section{STARSPOTS}

\subsection{Detection}

Fig. 5 shows the transit light curves after subtracting the best-fitting transit model from Section 3. The transits show many temporary brightenings that appear to be starspot occultation events.

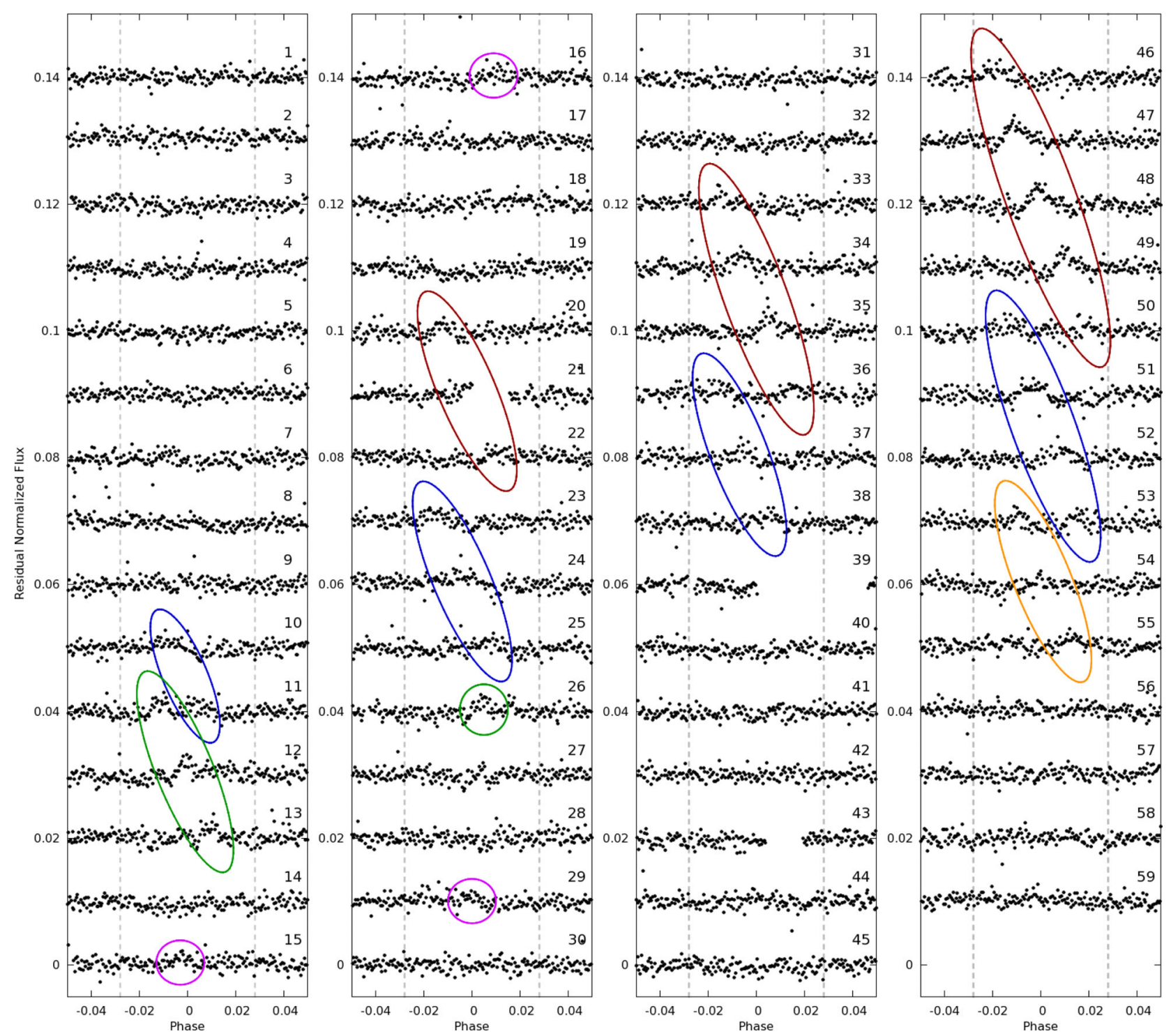

Figure 5. Starspot occultations in the model-subtracted light curve of Qatar-2b. Vertical dashed lines specify the extent of the transit. Sets of recurring occultations are marked with ellipses. Ellipses of the same colour correspond to the same starspot. 
We should first consider whether these could be residual artefacts from the $K 2$ thruster firings. However, this is highly unlikely since: (1) we know the times of thruster firings, and these do not coincide with the starspot features; (2) the starspots recur in relation to the planetary orbital and stellar rotation cycles, which the spacecraft firings do not; (3) the drift artefacts have a step-like appearance, which the starspot features do not; and (4) spacecraft drifts were particularly consistent during the observing Campaign 6 , which resulted in only minimal residual drift artefacts. Thus, we can conclude that the features are indeed occultations of starspots.

In order to identify starspot occultation events in an unbiased way, we shuffled the order of the transits in the residual light curve and then four of our colleagues examined them by eye and searched for any occultation-like features. First, we considered light curve features as starspot occultation candidates only if they were marked as a possible occultation event by at least two colleagues. We then fitted each occultation candidate with a Gaussian function to determine the orbital phase at which it occurs, and its amplitude and width, and calculated the corresponding change in the Bayesian information criterion (BIC). Finally, we report here only those occultation candidates whose BIC change was greater than 6 . These are marked with ellipses in Fig. 5 and listed in Table 2 along with stellar topocentric longitudes, which we calculated using the system parameters from Table 1 and the best-fitting occultation phases. Phase uncertainties in Table 2 are as quoted by the SciPy (Jones, Oliphant \& Peterson 2001) Gaussian fits, and stellar longitude uncertainties were calculated from the phase and system parameters uncertainties.

In total, we report the detection of 34 starspot occultation events, most of which appear at consistent phase shifts in up to five consecutive transits (transits 46-50). Furthermore, we found that sets of recurring occultations also appear at consistent phase shifts, in full agreement with the rotational modulation period (see Section 5) and the assumption that all recurring sets were produced by the same starspots. The occultation recurrence pattern and a very good fit to a single stellar rotational period (see Section 6.2) suggests that all the 34 individual starspot occultation events were caused by only five different starspots (each starspot is marked with a different colour in Fig. 5). In the longest recurring set of recurring occultations (transits 10-11, 23-25, 36-38 and 50-53), the starspot is seen during three full stellar rotations, spanning over 43 transits. This corresponds to a starspot lifetime of at least $58 \mathrm{~d}$. Fig. 5 also reveals that the shapes of recurring occultation events are changeable, indicative of starspot evolution.

Although the rotational modulation shown in Fig. 1 is produced by a contribution from several detected starspots and likely also by some unocculted and therefore undetected starspots, we found a tentative correlation between the main modulation components and individual detected starspots. The starspot marked blue in Fig. 5 is seen near mid-transit close to the main rotational modulation's minima, suggesting that this was then the largest among Qatar-2's starspots. The occultation events marked red in Fig. 5 cluster near the plateaus or secondary minima (seen clearest near BJD 2457244 and 2457282 in Fig. 1). On the other hand, the rotational modulation's maxima occur when no or only small starspot occultation events are detected.

\subsection{Rotational period}

The stellar rotational period can be calculated from the changes in starspot positions on the rotating stellar surface and the timing of the occultation events. The position of the starspot is defined by
Table 2. Phase and stellar topocentric longitude positions of every detected starspot occultation event. The change of BIC provides an estimate of each occultation's detectability.

\begin{tabular}{|c|c|c|c|}
\hline $\begin{array}{l}\text { Transit } \\
\text { number }\end{array}$ & Phase & $\begin{array}{c}\text { Stellar } \\
\text { longitude }{ }^{a}\left({ }^{\circ}\right)\end{array}$ & $\Delta \mathrm{BIC}$ \\
\hline 10 & $-0.0087 \pm 0.0012$ & $-21.1 \pm 3.0$ & 13.8 \\
\hline 11 & $-0.0112 \pm 0.0007$ & $-27.5 \pm 1.9$ & 15.2 \\
\hline 11 & $0.0027 \pm 0.0010$ & $6.4 \pm 2.4$ & 12.7 \\
\hline 12 & $-0.0006 \pm 0.0004$ & $-1.4 \pm 1.0$ & 28.8 \\
\hline 13 & $0.0098 \pm 0.0005$ & $23.9 \pm 1.3$ & 23.6 \\
\hline 15 & $-0.0025 \pm 0.0008$ & $-5.9 \pm 1.9$ & 17.7 \\
\hline 16 & $0.0102 \pm 0.0014$ & $24.9 \pm 3.7$ & 7.5 \\
\hline 20 & $-0.0128 \pm 0.0007$ & $-31.9 \pm 1.9$ & 9.8 \\
\hline 21 & $-0.0012 \pm 0.0027$ & $-2.8 \pm 6.4$ & 9.8 \\
\hline 22 & $0.0104 \pm 0.0012$ & $25.4 \pm 3.2$ & 9.3 \\
\hline 23 & $-0.0155 \pm 0.0008$ & $-39.8 \pm 2.4$ & 17.6 \\
\hline 24 & $-0.0049 \pm 0.0012$ & $-11.7 \pm 2.9$ & 27.9 \\
\hline 25 & $0.0070 \pm 0.0009$ & $16.8 \pm 2.2$ & 14.5 \\
\hline 26 & $0.0045 \pm 0.0005$ & $10.7 \pm 1.2$ & 20.8 \\
\hline 29 & $-0.0010 \pm 0.0012$ & $-2.4 \pm 2.8$ & 9.2 \\
\hline 33 & $-0.0151 \pm 0.0005$ & $-38.6 \pm 1.5$ & 16.4 \\
\hline 34 & $-0.0062 \pm 0.0006$ & $-14.8 \pm 1.5$ & 32.4 \\
\hline 35 & $0.0051 \pm 0.0003$ & $12.2 \pm 0.7$ & 42.1 \\
\hline 36 & $-0.0141 \pm 0.0011$ & $-35.6 \pm 3.1$ & 8.5 \\
\hline 36 & $0.0152 \pm 0.0008$ & $38.9 \pm 2.5$ & 11.3 \\
\hline 37 & $-0.0060 \pm 0.0008$ & $-14.3 \pm 2.0$ & 12.1 \\
\hline 38 & $0.0060 \pm 0.0007$ & $14.3 \pm 1.7$ & 9.0 \\
\hline 46 & $-0.0185 \pm 0.0011$ & $-49.8 \pm 3.9$ & 13.8 \\
\hline 47 & $-0.0106 \pm 0.0005$ & $-26.0 \pm 1.3$ & 46.1 \\
\hline 48 & $-0.0008 \pm 0.0004$ & $-1.9 \pm 1.0$ & 42.1 \\
\hline 49 & $0.0104 \pm 0.0010$ & $25.4 \pm 2.7$ & 36.2 \\
\hline 50 & $-0.0147 \pm 0.0020$ & $-37.4 \pm 5.7$ & 10.4 \\
\hline 50 & $0.0198 \pm 0.0011$ & $54.8 \pm 4.8$ & 7.3 \\
\hline 51 & $-0.0028 \pm 0.0010$ & $-6.6 \pm 2.4$ & 16.3 \\
\hline 52 & $0.0084 \pm 0.0007$ & $20.3 \pm 1.8$ & 17.6 \\
\hline 53 & $-0.0096 \pm 0.0005$ & $-23.4 \pm 1.3$ & 9.9 \\
\hline 53 & $0.0150 \pm 0.0012$ & $38.3 \pm 3.7$ & 6.0 \\
\hline 54 & $0.0001 \pm 0.0010$ & $0.2 \pm 2.4$ & 8.4 \\
\hline 55 & $0.0130 \pm 0.0010$ & $32.5 \pm 2.9$ & 23.5 \\
\hline
\end{tabular}

Note. ${ }^{a}$ Stellar topocentric longitude runs from $-90^{\circ}$ (first planetary contact), through $0^{\circ}$ (central meridian) to $90^{\circ}$ (last contact).

stellar longitude and latitude and, in general, both parameters are needed for accurate determination of the starspot's position changes. However, in aligned systems such as Qatar-2 (see Section 6.3) and in the presence of recurring sets of recurring occultations, the most relevant starspot parameter is longitude.

Fig. 6 shows the measured phase and longitude positions of all the occultation events from Table 2. Using the best-fitting longitude versus phase dependence for every recurring set, and the known orbital period of the planet, we were able to calculate the rotational period for every starspot. Their uncertainties were calculated from the slope uncertainties quoted by the SciPy fitting routine. The resulting rotational periods and their uncertainties are given in Table 3 and are based on the assumptions that starspots have circular shapes and that the starspots' positions change only due to stellar rotation, i.e. that starspot longitudinal migration is negligible. The latter assumption is justified by the solar observations, which reveal that the longitudinal migration of sunspots is four orders of magnitude smaller than the solar rotation rate (Gyenge, Baranyi \& Ludmány 2014). The weighted mean rotational period of $18.0 \pm 0.2 \mathrm{~d}$ is in agreement with the rotational modulation period (see Section 5). We also performed a simultaneous fit of all 


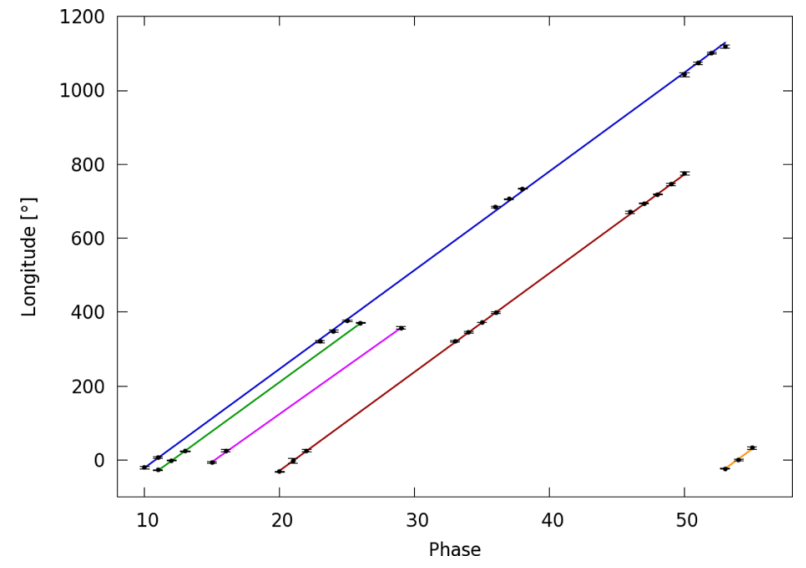

Figure 6. Longitude and phase positions of all recurring occultations. We added $360^{\circ}$ to stellar longitudes from Table 2 for every stellar rotation. Each best-fitting straight line corresponds to a different starspot, which are marked with the same colours as in Fig. 5. The good fits to straight lines of very similar slopes suggest that occultation events of each recurring set were very likely caused by the same starspot. A few outliers and weak skewing trends, such as in the recurring set 50-53, may be attributed to non-circular and evolving starspot shapes, supported by the variable and non-Gaussian appearance of occultation events in Fig. 5.

Table 3. Stellar rotational period of every starspot.

\begin{tabular}{lc}
\hline Transit numbers & Rotational period $(\mathrm{d})$ \\
\hline $10-11+23-25+36-38+50-53$ & $18.00 \pm 0.03$ \\
$11-13+26$ & $18.11 \pm 0.07$ \\
$15-16+29$ & $18.58 \pm 0.17$ \\
$20-22+33-36+46-50$ & $18.01 \pm 0.04$ \\
$53-55$ & $18.00 \pm 0.97$ \\
weighted mean & $\mathbf{1 8 . 0 3} \pm \mathbf{0 . 2 4}$ \\
\hline
\end{tabular}

recurring sets with a common rotational period and obtained the same result as a weighted mean. Because rotational modulations depend strongly on starspots coming and going, which causes modulational phase shifts, the period derived from starspot occultation events is more accurate and reliable than the period determined from the rotational modulation.

The recurring set of starspot occultations during transits 15-16 and 29 indicated a $0.5 \mathrm{~d}(2.3 \sigma)$ longer rotational period than the weighted mean (see Table 3 ), possibly caused by a differential rotation. However, we were unable to investigate the effect of differential rotation due to the relatively small differences in rotational periods among different spots and because of poorly constrained starspot latitudes (see Section 6.3).

Our rotational period disagrees with the previously reported value of $14.8 \pm 0.3 \mathrm{~d}$ by Mancini et al. $(2014,2016)$. Their rotational period was based on two starspot occultation events separated by $12 \mathrm{~d}$, which were consistent with the projected rotational velocity of $2.8 \pm 0.5 \mathrm{~km} \mathrm{~s}^{-1}$ by Bryan et al. (2012). With our robust rotational period of $18.0 \pm 0.2 \mathrm{~d}$ it now seems reasonable to conclude that the two starspot occultation events analysed by Mancini et al. (2014, 2016) (spots \#1 and \#2) were in fact caused by two different spots and that the projected rotational velocity by Bryan et al. (2012) was overestimated by at least $1.5 \sigma$. A slower rotation than previously thought is further supported by the latest measurements of projected rotational velocity which place it at $2.0 \pm 1.0 \mathrm{~km} \mathrm{~s}^{-1}$ (Esposito et al. 2017).

\subsection{Obliquity}

The angle between the stellar rotational axis and the planetary orbital axis results in a gradual latitudinal positional drift of the recurring starspot occultation events. In misaligned systems, the starspot occultations may occur only at preferential transit phases where the active latitudinal regions cross the planetary transit chord, such as in the HAT-P-11 system (Sanchis-Ojeda \& Winn 2011). In the Qatar-2 system, the starspot occultation events occur uniformly along the transit chord and in consecutive transits (see Fig. 5), which indicates that the system is aligned, as in the case of the Kepler-17 system (Désert et al. 2011).

We fitted the starspot occultation events with the Planetary Retrospective Integrated Starspot Model (PRISM; Tregloan-Reed, Southworth \& Tappert 2013; Tregloan-Reed et al. 2015) and searched for any gradual starspot latitudinal drifts. We used the fixed input system parameters from Section 3 and searched for best-fitting starspot topocentric longitude, latitude, radius and contrast. We explored the entire starspot parameter space for each occultation event in each set of recurring occultations. Since PRISM was designed to model starspots with circular shapes, some of the occultation events in the Qatar-2 light curve were fitted poorly owing to their non-Gaussian shapes (e.g. set 50-53 indicated that the spot was elongated along the transit chord, possibly a strip of spots). We also found a high degree of degeneracy and non-physically rapid and non-monotonic starspot parameter variations among consecutive occultation events within several sets. Therefore, we decided to find the starspot radius and contrast that gave the best and most consistent fit within each set of consecutively recurring occultations and kept them fixed while refitting for starspot longitude and latitude positions. By using this approach we ignore starspot evolution, which may be correct only for time-scales significantly shorter than starspot lifetimes, and we assume that starspot contrast does not change as a function of the spot's distance from the stellar disc centre. The latter assumption is justified by the findings of Lanza et al. (2003), who calculated that sunspot contrast changes only by a few per cent from the solar disc centre to the limb. Having the starspot radius and contrast fixed, the best-fitting latitudes could be determined from the widths and heights of each of the occultation events within the same set. This approach lifted the degeneracy and provided a physically more plausible solution.

In this paper, we present only the obliquity results that were derived from the longest individual set of consecutively recurring occultations (transits 46-50), which yielded the most robust obliquity measurement with the smallest uncertainty. First, this set exhibited pronounced Gaussian-like occultation events that enabled a reliable starspot parameter fitting. And secondly, by exhibiting five occultation events in consecutive transits it provided the largest longitudinal starspot coverage in the shortest possible time-span of $5 \mathrm{~d}$, which is essential when assuming no starspot evolution.

The light curves and visualizations of the best-fitting starspot models for transits 46-50 are shown in Fig. 7. Starspot position parameters are listed in Table 4. The stellar longitude and latitude uncertainties in Table 4 are $1 \sigma$ uncertainties as given by PRISM, which estimates uncertainties from the distribution of starspot parameters in the MCMC chain. We show the starspot longitudinal and latitudinal positions in Fig. 8 and find that they change linearly. If the stellar inclination was significant, the starspots' path would act as a quadratic function. We find no evidence for non-linear starspot paths, which indicates that the stellar rotation must be close to edge-on. Using the system parameters from Section 3 and the fitted relation between the starspot longitudinal and 

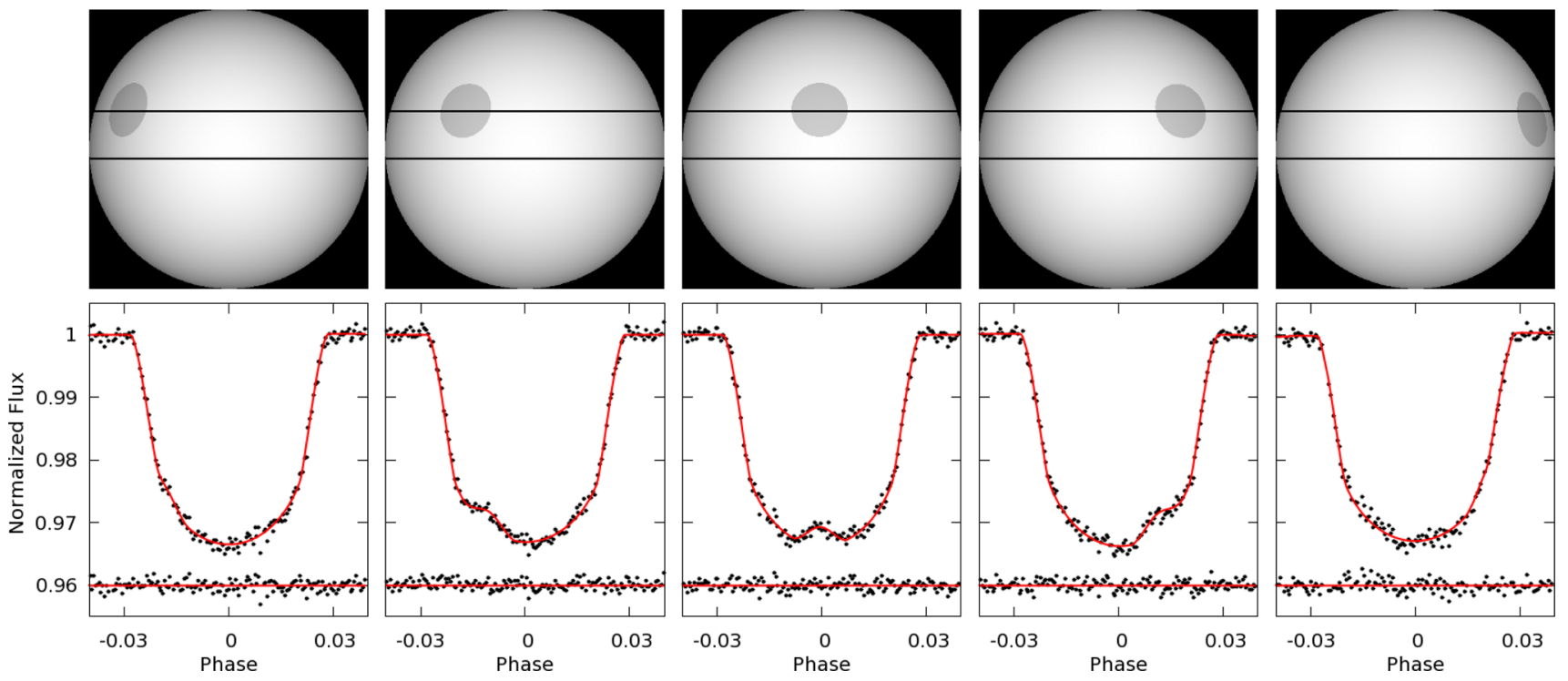

Figure 7. Best-fitting starspot models for transits 46-50. Upper panels: starspot visualizations. Horizontal lines indicate the position of the transit chord. Lower panels: corresponding best-fitting light curve models. Shown below are the residual light curves after subtracting the starspot models. A notable residual in transit 50 at phase -0.015 is caused by another starspot (see Table 2 and Fig. 5).

Table 4. Best-fitting starspot positions with PRISM for the longest individual set of recurring occultations, using the fixed starspot angular radius of $11.6^{\circ} \pm 2.5^{\circ} a$ and contrast of $0.810 \pm 0.058^{b}$.

\begin{tabular}{ccc}
\hline $\begin{array}{c}\text { Transit } \\
\text { number }\end{array}$ & $\begin{array}{c}\text { Stellar } \\
\text { longitude }^{c}\left({ }^{\circ}\right)\end{array}$ & $\begin{array}{c}\text { Stellar } \\
\text { latitude }^{d}\left({ }^{\circ}\right)\end{array}$ \\
\hline 46 & $-50.1 \pm 2.4$ & $16.51 \pm 0.99$ \\
47 & $-26.8 \pm 1.1$ & $16.15 \pm 0.66$ \\
48 & $-0.99 \pm 0.80$ & $16.55 \pm 0.73$ \\
49 & $27.86 \pm 0.94$ & $16.08 \pm 0.64$ \\
50 & $60.2 \pm 2.1$ & $12.5 \pm 6.6$ \\
\hline
\end{tabular}

Notes. ${ }^{a}$ Angular radius runs from 0 to $90^{\circ}$, where $90^{\circ}$ covers half of the stellar surface. An angular radius of $11.6^{\circ} \pm 2.5^{\circ}$ corresponds to an actual radius of $(9.9 \pm 2.1) \times 10^{4} \mathrm{~km}$.

${ }^{b}$ Contrast runs from 0 to 1 , where a value of 1 corresponds to the brightness of the surrounding photosphere.

${ }^{c}$ Longitude runs from $-90^{\circ}$ (first planetary contact), through $0^{\circ}$ (central meridian) to $90^{\circ}$ (last contact).

${ }^{d}$ Latitude runs from $-90^{\circ}$ (stellar south pole), through

$0^{\circ}$ (equator) to $90^{\circ}$ (north pole).

latitudinal positions from Fig. 8, we derive the sky-projected obliquity of $-0.2^{\circ} \pm 0.7^{\circ}$. For a degenerate but less-likely scenario in which a starspot appears below the transit chord, we obtain a similar sky-projected obliquity of $0.1^{\circ} \pm 0.8^{\circ}$. Both calculations are based on the assumption that the starspot's size and contrast do not change throughout the set and that the starspot's latitudinal position only changes due to obliquity, i.e. neglects the contribution of latitudinal starspot migration. Therefore, the uncertainty is likely to be underestimated. In a very conservative approach of Désert et al. (2011), we set the upper uncertainty limit to $8^{\circ}$, which is the largest possible sky-projected obliquity to still be able to see occultation events in five consecutive transits of Qatar-2b. The realistic uncertainty is expected to lie somewhere in between the two given values. However, because we were unable to quantify all the systematic errors and realistic ranges of starspot contrast and radius variability, we adopt $8^{\circ}$ as the final conservative uncertainty. A similar obliquity

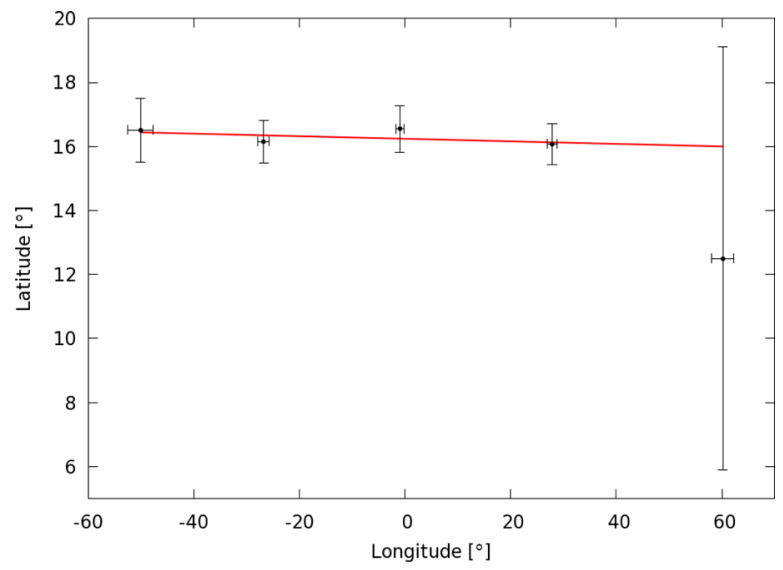

Figure 8. Latitude and longitude positions for starspots in transits 46-50. The fit corresponds to the sky-projected obliquity angle of $-0.2^{\circ} \pm 0.7^{\circ}$. The large uncertainty of the right most data point (transit 50) is because of the starspot's proximity to the stellar limb and a low signal-to-noise ratio of the occultation event.

constraint was provided by Dai et al. (2017), who claim that Qatar2's sky-projected obliquity is smaller than $10^{\circ}$.

The small obliquity angle agrees with the empirical indication that systems with host stars cooler than $6250 \mathrm{~K}$ are generally aligned (Winn et al. 2010; Albrecht et al. 2012).

\section{PHASE-CURVE MODULATION}

The three main phase-curve modulation components of planetary systems in the optical wavelengths covered by the $K 2$ bandpass (420-900 nm) are as follows: (1) reflection of starlight from an orbiting planet; (2) rotation of the star that is gravitationally distorted into an ellipsoidal shape; and (3) Doppler beaming of starlight by the orbital motion of the host star (Esteves, De Mooij \& Jayawardhana 2013). To search for these phase-curve modulation components, we first removed the rotational modulation and normalized the $K 2$ light curve by dividing it with the median of 


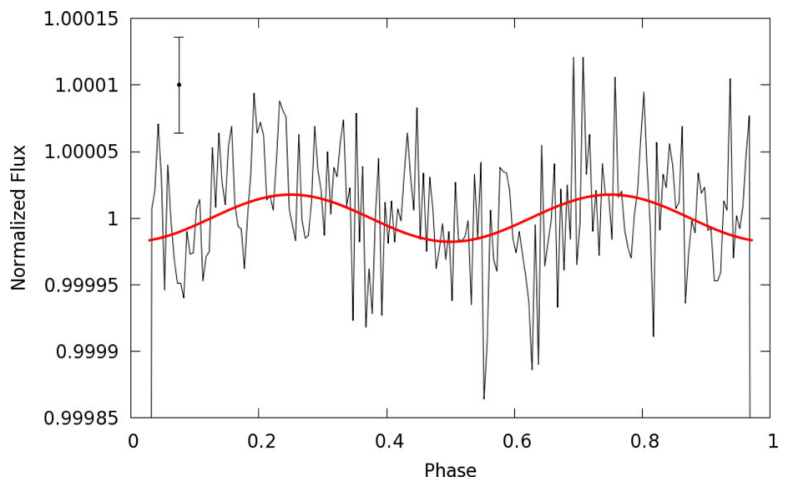

Figure 9. Phase-curve of Qatar-2, binned to 200 bins. The red line denotes a possible detection of the ellipsoidal modulation with the best-fitting semiamplitude of $18 \pm 4 \mathrm{ppm}$. Rotational and Doppler beaming modulation components are not significantly detected. One representative error bar is shown in the top left-hand corner.

second-order polynomial fits with a window and step size of 3 and $0.3 \mathrm{~d}$, respectively. Using this approach, we effectively removed the low-frequency rotational modulation while retaining any potential high-frequency phase-curve modulation, as confirmed by successful phase-curve signal injection and recovery tests. Next, we phase-folded the normalized light curve on the orbital period of Qatar-2b and applied binning to reduce the white noise (Fig. 9).

Using the system parameters from Table 1, the theoretically expected semi-amplitudes of the reflection, ellipsoidal and Doppler beaming phase-curve components are $595 A_{\mathrm{g}} \mathrm{ppm}, 12 \mathrm{ppm}$ and $8 \mathrm{ppm}$, respectively, where $A_{\mathrm{g}}$ is the planet's geometrical albedo (Mazeh \& Faigler 2010). We tentatively detect a sinusoidal signal peaking at orbital phases 0.25 and 0.75 with a minimum at phase 0.5 , characteristic of ellipsoidal modulations. Its best-fitting semiamplitude of $18 \pm 4 \mathrm{ppm}$ is close to the theoretically expected value. We were able to detect this modulation with a similar amplitude in the whole, first half and second half of the light curve, and also using three additional artefact removal procedures (K2SFF, K2SC and EVEREST). This strengthens the assumption that the detection of ellipsoidal modulation is real. However, the fit improves the reduced $\chi^{2}$ only slightly from 1.62 to 1.50 . The light curve exhibits significant red noise such as SFF artefacts or residuals of the imperfect removal of the pronounced rotational modulation, and therefore this potential detection of ellipsoidal modulation should be regarded as tentative.

A reflectional modulation would peak at phase 0.5. A simultaneous MCMC fit of all three possible phase-curve modulation components gives the best-fitting reflectional modulation semi-amplitude of $2 \mathrm{ppm}$, though given the presence of red noise a conservative upper limit would be much higher, at $30 \mathrm{ppm}$. This reflection modulation upper limit of $30 \mathrm{ppm}$ implies that the planet's optical geometric albedo has to be lower than 0.05. Low albedos at optical wavelengths are common for hot Jupiters (Esteves et al. 2013), and are consistent with theoretical models that predict cloudless planetary atmospheres (Burrows, Ibgui \& Hubeny 2008). The lowest optical geometric albedo has been measured for the TrES-2 system of less than 0.01 (Kipping \& Bakos 2011).

As of the time of writing, the phase-curve modulations have been detected for 26 planetary systems, 19 of which are from Kepler observations according to the NASA Exoplanet Archive. ${ }^{2}$ If our

\footnotetext{
${ }^{2}$ http://exoplanetarchive.ipac.caltech.edu/
}

possible detection of ellipsoidal modulation is real, it would be the first phase-curve modulation detection of any of the planetary systems observed by $K 2$.

\section{NO ADDITIONAL TRANSITING PLANETS}

We searched for any additional transiting planets in the normalized K2 light curve with low-frequency modulations removed. First, we removed Qatar-2b's transits from the light curve by replacing the measured normalized flux values around transits with unity. We then produced the periodogram to search for any remaining periodic transit-like features with PyKE tool KEPBLS, which is based on the box-least-square fitting algorithm by Kovács, Zucker $\&$ Mazeh (2002). The periodogram did not reveal any significant peaks between 0.5 and $30 \mathrm{~d}$ period with a transit depth upper limit of $280 \mathrm{ppm}$.

\section{AGE OF THE HOST STAR}

We estimate the stellar age first from the rotational period. Young stars rotate rapidly and gradually slow down as they lose angular momentum through magnetized stellar winds (Barnes 2003). We used the gyrochronological relation by Barnes (2007) to estimate that Qatar-2 is $0.59 \pm 0.10$ Gyr old.

Comparing stellar parameters to theoretical stellar models provides another age estimate. As in Močnik et al. (2016a), we compared stellar density and effective temperature of $4645 \mathrm{~K}$ to isochrones and evaluated the age of the star using the Bayesian mass and age estimator BAGEMASS (Maxted, Serenelli \& Southworth 2015a). The stellar evolution models used in BAGEMASS were calculated using the GARSTEC code (Weiss \& Schlattl 2008). The best-fitting stellar evolution track gives an age estimate of $9.4 \pm 3.2 \mathrm{Gyr}$.

The huge discrepancy between gyrochronological and isochronal age estimates has also been seen in other exoplanet host stars, in particular, K-type stars hosting a hot Jupiter (Maxted, Serenelli \& Southworth 2015b; Mancini et al. 2017). It has been suggested that these discrepancies could be caused by the tidal interactions, which could transfer angular momentum from an orbital motion of a planet to the rotation of a host star (Maxted et al. 2015b). Thus, Qatar-2 may have been spun-up by the hot Jupiter and it is actually older than the gyrochronological age estimate. Another explanation for the age discrepancy is the radius anomaly of late type stars, where the observed stellar radii are larger than stellar model predictions (Popper 1997), which could result in isochronal ages being overestimated.

Obtaining a reliable age estimate for Qatar-2 would require additional age estimators such as the photospheric $\mathrm{Li}$ abundance, or an in-depth modelling to account for the two above-mentioned mechanisms that are probably contributing to the age discrepancy.

\section{CONCLUSIONS}

We used the $K 2$ short-cadence observations of Qatar-2 from observing Campaign 6 to refine the system parameters, search for TTVs, additional transiting planets, phase-curve modulations, and mainly to analyse the starspot activity.

The light curve of Qatar-2 exhibits pronounced rotational modulation with a period of $18.2 \pm 1.6 \mathrm{~d}$ and an amplitude of about 2 per cent. The stellar activity is further manifested through 34 detected starspot occultation events among 59 transits. The majority of the occultations are seen repeatedly in up to five consecutive 
transits, owing to the low obliquity angle and a large ratio between stellar rotational and planet's orbital period. Moreover, sets of recurring occultations also reappear after several stellar rotations. The longest recurring set of recurring starspot occultations spans over three stellar rotations, which sets the longest starspot lifetime to at least $58 \mathrm{~d}$. By fitting the recurring occultation events, we derived the stellar rotational period of $18.0 \pm 0.2 \mathrm{~d}$, in agreement with the rotational modulation period, and zero sky-projected obliquity with a conservative error bar of $\pm 8^{\circ}$.

We detect a possible ellipsoidal phase-curve modulation with a semi-amplitude of $18 \pm 4 \mathrm{ppm}$, close to the theoretically expected value. Due to the presence of red noise this possible detection of ellipsoidal modulation should be regarded as tentative.

\section{ACKNOWLEDGEMENTS}

We thank the anonymous referees for their perceptive comments that led to improving this paper. We gratefully acknowledge the financial support from the Science and Technology Facilities Council, under grants ST/J001384/1, ST/M001040/1 and ST/M50354X/1. This paper includes data collected by the $K 2$ mission. Funding for the $K 2$ mission is provided by the NASA Science Mission directorate. This work made use of PукE (Still \& Barclay 2012), a software package for the reduction and analysis of Kepler data. This open source software project is developed and distributed by the NASA Kepler Guest Observer Office. This research has made use of the NASA Exoplanet Archive, which is operated by the California Institute of Technology, under contract with the National Aeronautics and Space Administration under the Exoplanet Exploration Program.

\section{REFERENCES}

Agol E., Steffen J., Sari R., Clarkson W., 2005, MNRAS, 359, 567 Aigrain S., Parviainen H., Pope B. J. S., 2016, MNRAS, 459, 2408 Albrecht S. et al., 2012, ApJ, 757, 18

Anderson D. R. et al., 2012, MNRAS, 422, 1988

Anderson D. R. et al., 2015, A\&A, 575, A61

Barnes S. A., 2003, ApJ, 586, 464

Barnes S. A., 2007, ApJ, 669, 1167

Borucki W. J. et al., 2010, Science, 327, 977

Bradshaw S. J., Hartigan P., 2014, ApJ, 795, 79

Bryan M. L. et al., 2012, ApJ, 750, 84

Bryan M. L. et al., 2014, ApJ, 782, 121

Burrows A., Ibgui L., Hubeny I., 2008, ApJ, 682, 1277

Claret A., 2000, A\&A, 363, 1081

Claret A., 2004, A\&A, 428, 1001

Collier Cameron A. et al., 2007, MNRAS, 380, 1230

Collier Cameron A., Bruce V. A., Miller G. R. M., Triaud A. H. M. J., Queloz D., 2010, MNRAS, 403, 151

Dai F., Winn J. N., Yu L., Albrecht S., 2017, AJ, 153, 40

Désert J.-M. et al., 2011, ApJS, 197, 14

Esposito M. et al., 2017, A\&A, 601, A53

Esteves L. J., De Mooij E. J. W., Jayawardhana R., 2013, ApJ, 772, 51
Gaudi B. S., Winn J. N., 2007, ApJ, 655, 550

Gyenge N., Baranyi T., Ludmány A., 2014, Sol. Phys., 289, 579

Howell S. B. et al., 2014, PASP, 126, 398

Jones E., Oliphant T., Peterson P., 2001, SciPy: Open Source Scientific Tools for Python, Available at http://www.scipy.org/

Kipping D., Bakos G., 2011, ApJ, 733, 36

Kovács G., Zucker S., Mazeh T., 2002, A\&A, 391, 369

Lanza A. F., Rodonò M., Pagano I., Barge P., Llebaria A., 2003, A\&A, 403, 1135

Luger R., Agol E., Kruse E., Barnes R., Becker A., Foreman-Mackey D., Deming D., 2016, AJ, 152, 100

Mancini L. et al., 2014, MNRAS, 443, 2391

Mancini L. et al., 2016, MNRAS, 462, 4266

Mancini L. et al., 2017, MNRAS, 465, 843

Maxted P. F. L., Serenelli A. M., Southworth J., 2015a, A\&A, 575, A36

Maxted P. F. L., Serenelli A. M., Southworth J., 2015b, A\&A, 577, A90

Mayor M., Queloz D., 1995, Nature, 378, 355

Mazeh T., Faigler S., 2010, A\&A, 521, L59

Mazeh T. et al., 2013, ApJS, 208, 16

McIntosh P. S., 1981, in Cram L. E., Thomas J. H., eds, The Physics of Sunspots. Sacramento Peak National Observatory, Sunspot, New Mexico, p. 7

Močnik T. et al., 2016a, PASP, 128, 124403

Močnik T., Clark B. J. M., Anderson D. R., Hellier C., Brown D. J. A., 2016b, AJ, 151, 150

Nesvorný D., Kipping D., Terrell D., Hartman J., Bakos G. Á., Buchhave L. A., 2013, ApJ, 777, 3

Oshagh M., Santos N. C., Boisse I., Boué G., Montalto M., Dumusque X., Haghighipour N., 2013, A\&A, 556, A19

Pollacco D. et al., 2008, MNRAS, 385, 1576

Popper D. M., 1997, AJ, 114, 1195

Sanchis-Ojeda R., Winn J. N., 2011, ApJ, 743, 61

Silva A. V. R., 2003, ApJ, 585, L147

Sing D. K., 2010, A\&A, 510, A21

Solanki S. K., 2003, A\&A Rev., 11, 153

Southworth J., 2011, MNRAS, 417, 2166

Still M., Barclay T., 2012, PyKE: Reduction and Analysis of Kepler Simple Aperture Photometry Data, Astrophysics Source Code Library, record ascl:1208.004

Tregloan-Reed J., Southworth J., Tappert C., 2013, MNRAS, 428, 3671

Tregloan-Reed J. et al., 2015, MNRAS, 450, 1760

Vanderburg A., Johnson J. A., 2014, PASP, 126, 948

Weiss A., Schlattl H., 2008, Ap\&SS, 316, 99

Winn J. N. et al., 2010, ApJ, 723, L223

\section{APPENDIX A: BARYCENTRIC-CORRECTED RADIAL VELOCITIES}

Bryan et al. (2012) published 44 Qatar-2 radial velocity (RV) measurements which they obtained with TRES between 2011 January 18 to June 21. As pointed out in their erratum (Bryan et al. 2014), the data reduction pipeline that they used to calculate the RVs had a bug, which parsed Qatar-2's negative declination with positive arcminutes and arcseconds for the barycentric correction. However, they did not provide the corrected RVs in their erratum. Table A1 lists TRES RVs of Qatar-2 with correct barycentric correction. 
Table A1. TRES barycentric-corrected RVs for Qatar-2. The RVs are given relative to the RV measured on BJD 2455646.85 .

\begin{tabular}{|c|c|c|}
\hline BJD - 2450000 & $\mathrm{RV}\left(\mathrm{km} \mathrm{s}^{-1}\right)$ & $\Delta \mathrm{RV}\left(\mathrm{km} \mathrm{s}^{-1}\right)$ \\
\hline 5580.011622 & 0.001 & 0.034 \\
\hline 5581.027117 & 0.158 & 0.038 \\
\hline 5583.034587 & -0.796 & 0.027 \\
\hline 5583.961503 & 0.066 & 0.033 \\
\hline 5585.003598 & 0.123 & 0.027 \\
\hline 5587.983438 & 0.082 & 0.027 \\
\hline 5602.958910 & -0.516 & 0.033 \\
\hline 5604.034894 & 0.097 & 0.030 \\
\hline 5604.966229 & -0.051 & 0.029 \\
\hline 5605.966418 & -0.844 & 0.024 \\
\hline 5607.001348 & -0.638 & 0.033 \\
\hline 5607.987854 & 0.249 & 0.036 \\
\hline 5608.955271 & -0.188 & 0.028 \\
\hline 5610.996605 & -0.533 & 0.037 \\
\hline 5615.938163 & 0.217 & 0.024 \\
\hline 5616.973605 & -0.136 & 0.029 \\
\hline 5617.978176 & -0.860 & 0.021 \\
\hline 5643.881697 & 0.211 & 0.029 \\
\hline 5644.849974 & -0.641 & 0.026 \\
\hline 5645.901592 & -0.803 & 0.021 \\
\hline 5646.846694 & 0.000 & 0.017 \\
\hline 5647.890953 & 0.162 & 0.035 \\
\hline 5650.857343 & 0.060 & 0.027 \\
\hline 5652.895515 & -0.599 & 0.033 \\
\hline 5656.870962 & -0.706 & 0.022 \\
\hline 5659.877783 & 0.124 & 0.025 \\
\hline 5662.839965 & 0.174 & 0.029 \\
\hline 5663.812865 & -0.081 & 0.021 \\
\hline 5664.867650 & -0.763 & 0.030 \\
\hline 5665.796571 & -0.549 & 0.021 \\
\hline 5668.845188 & -0.838 & 0.030 \\
\hline 5671.762510 & -0.218 & 0.022 \\
\hline 5672.733966 & -0.858 & 0.029 \\
\hline 5673.784772 & -0.480 & 0.019 \\
\hline 5691.770974 & -0.340 & 0.032 \\
\hline 5702.699240 & 0.146 & 0.024 \\
\hline 5703.727260 & -0.559 & 0.032 \\
\hline 5704.730193 & -0.816 & 0.020 \\
\hline 5705.694921 & 0.054 & 0.042 \\
\hline 5706.738098 & 0.160 & 0.028 \\
\hline 5722.744329 & 0.168 & 0.034 \\
\hline 5726.718494 & 0.085 & 0.030 \\
\hline 5728.755461 & -0.695 & 0.032 \\
\hline 5733.706680 & 0.189 & 0.028 \\
\hline
\end{tabular}

This paper has been typeset from a $\mathrm{T}_{\mathrm{E}} \mathrm{X} / \mathrm{LT} \mathrm{E} \mathrm{X}$ file prepared by the author. 